\title{
Le succès des " héritières » : effet conjugué du genre et du niveau d'études des parents sur la réussite à l'université
}

The success of "héritières" (female inheritors): Joint impact of gender and parents' level of education on achievement at university El éxito de las «herederas»: Efecto conjugado del tipo y del nivel de estudios de los padres en el éxito en la carrera universitaria Der Erfolg der „Erben“: Zusammenspiel des Genders und des Ausbildungsniveaus der Eltern auf den Erfolg an der Universität

Dominique Lafontaine, Vincent Dupriez, Maud Van Campenhoudt et Catherine Vermandele

\section{(2) OpenEdition}

\section{Journals}

Édition électronique

URL : http://journals.openedition.org/rfp/3656

DOI : $10.4000 /$ rfp.3656

ISSN : 2105-2913

Éditeur

ENS Éditions

\section{Édition imprimée}

Date de publication : 15 juin 2012

Pagination : $29-48$

ISBN : 978-2-84788-379-4

ISSN : 0556-7807

Référence électronique

Dominique Lafontaine, Vincent Dupriez, Maud Van Campenhoudt et Catherine Vermandele, «Le succès des « héritières » : effet conjugué du genre et du niveau d'études des parents sur la réussite à l'université », Revue française de pédagogie [En ligne], 179 | avril-juin 2012, mis en ligne le 15 juin 2015, consulté le 20 avril 2019. URL : http://journals.openedition.org/rfp/3656 ; DOI : 10.4000/ rfp.3656 


\section{Le succès des « héritières »: effet conjugué du genre et du niveau d'études des parents sur la réussite à l'université Dominique Lafontaine, Vincent Dupriez,
Maud Van Campenhoudt et Catherine Vermandele}

Cette recherche porte sur les facteurs sociodémographiques et les parcours scolaires antérieurs liés à la réussite de la première année d'université. L'analyse de données empiriques collectées en Belgique francophone met en évidence une meilleure réussite des filles, qui ne s'explique pas par les caractéristiques du parcours scolaire antérieur. Au delà de cette différence de réussite selon le sexe, des effets d'interaction entre le sexe et le niveau d'études des parents se font jour. Ainsi, les écarts de réussite selon le sexe, dans tous les cas en faveur des filles, se creusent à mesure que le diplôme des parents s'élève. Par ailleurs, à diplôme du père équivalent, le diplôme de la mère n'explique pas la réussite des garçons, mais est significativement lié à la réussite des filles.

Mots-clés (TESE) : résultats de l'éducation, sexe, environnement socioculturel, inégalité sociale, égalité des sexes, parents.

\section{INTRODUCTION}

L'enseignement supérieur dans les pays économiquement avancés a connu au cours des dernières décennies une expansion sans précédent. Cette expansion quantitative s'est accompagnée dans presque tous ces pays d'une différenciation des cursus. Ainsi, selon Shavit, Arum et Gamoran (2007), " les systèmes qui se composaient quasi exclusivement d'universités de recherche ont développé des instituts d'enseignement supérieur de second rang, moins sélectifs, et l'essentiel de la croissance du nombre d'étudiants a été absorbé dans ces institutions de second rang. Ainsi, alors que les membres de la classe ouvrière gagnaient de nouvelles occasions d'entrer dans l'enseignement supérieur, le système s'est hiérarchiquement différencié, en sorte que les occasions qui s'ouvraient peuvent avoir perdu en valeur » (Shavit, Arum \& Gamoran, 2007, p. 1). Comme le soulignent ces mêmes auteurs, les modes de différenciation varient selon le système éducatif et il est donc impérieux d'aborder la manière dont se tisse la question des enjeux de démocratisation dans différents contextes nationaux, qui ont chacun leurs spécificités. C'est l'ambition du présent article. II s'agit d'étudier de façon empirique la question des inégalités de réussite selon le sexe et le niveau de diplôme des parents, en Communauté française de Belgique (CFB). Dans un premier temps, le lien entre le sexe et la réussite de la première année d'université sera étudié, en tenant sous contrôle le parcours scolaire antérieur ; dans un deuxième temps, on étudiera plus avant les effets d'interaction entre le sexe de l'étudiant et le niveau d'études de ses parents. 


\section{L'ÉTAT DE LA QUESTION}

\section{Réussite selon le sexe}

Toutes les études menées au niveau international (OCDE, 2010 ; Eurydice, 2007) montrent une meilleure réussite des filles au niveau universitaire et de meilleurs taux de diplomation, qui progressent plus vite que ceux des garçons au cours de la dernière décennie (Eurydice, 2007 ; Buchmann, DiPrete \& McDaniel, 2008). Comme l'indiquent Shavit, Arum et Gamoran (2007), « l'avantage des garçons en termes d'accomplissements éducatifs a dramatiquement diminué au cours de la seconde moitié du xxe siècle [...]. Les données montrent une augmentation moyenne de l'écart en fonction du sexe " (Shavit, Arum \& Gamoran, 2007, p. 27). La CFB ne fait pas exception à cet égard : toutes les recherches menées mettent clairement en évidence que le taux de réussite des filles est supérieur à celui des garçons, non seulement en première année à l'université, mais aussi tout au long du cursus universitaire, jusqu'à l'obtention d'un diplôme. Ainsi, pour l'année académique 2008-
2009, le taux de réussite en première année d'études à l'université des étudiants de première génération ${ }^{1}$ est de $41 \%$ chez les filles alors qu'il s'élève à $34 \%$ chez les garçons. Par ailleurs, les filles ont un taux de réussite supérieur à celui des garçons, dans pratiquement tous les domaines d'études (données du $\mathrm{CREF}^{2}$, voir CREF, 2003). Le taux d'obtention d'un diplôme du premier cycle est légèrement supérieur chez les filles : l'analyse des cohortes des étudiants de première génération des années académiques 1995-1996 et 1996-1997 montre que $55 \%$ des filles entrées à l'université dans un premier cycle d'une durée théorique de deux ans ont obtenu un diplôme, contre $48 \%$ des garçons (Droesbeke, Lecrenier, Tabutin et al., 2008, p. 123). Cette même analyse met également en évidence que, dans tous les domaines d'études, le pourcentage de diplômés du premier cycle qui ont répété au moins une année avant l'obtention de leur diplôme est plus élevé chez les garçons que chez les filles (Droesbeke, Lecrenier, Tabutin et al., 2008, p. 138).

Mais pourquoi les filles réussissent-elles mieux à l'université que les garçons ? Une des difficultés

\section{Caractéristiques de l'enseignement secondaire et accès à l'enseignement supérieur}

En Communauté française de Belgique, la fréquentation de l'enseignement secondaire est obligatoire jusqu'à l'âge de 18 ans. Les six années d'études de l'enseignement secondaire, auquel accèdent normalement les élèves à l'âge de 12 ans, se subdivisent en trois degrés : le $1^{\mathrm{er}}$ degré (les $1^{\mathrm{re}}$ et $2^{\mathrm{e}}$ années), le $2^{\mathrm{e}}$ degré (les $3^{e}$ et $4^{\mathrm{e}}$ années) et le $3^{\mathrm{e}}$ degré (les $5^{\mathrm{e}}$ et $6^{\mathrm{e}}$ années). Au $1^{\text {er }}$ degré, la très grande majorité des élèves suivent une formation commune. À partir de la $3^{e}$ année du secondaire, les élèves ont le choix entre deux grandes filières d'enseignement :

- l'enseignement de transition, qui prépare et donne accès à l'enseignement supérieur. On y distingue l'enseignement général et l'enseignement technique ou artistique de transition ;

- l'enseignement de qualification, dont la finalité première est de préparer à un métier. On y distingue l'enseignement technique ou artistique de qualification et l'enseignement professionnel.

Les taux de redoublement dans l'enseignement secondaire sont élevés ; en 2009-2010, seuls quatre jeunes sur dix sont à l'heure dans leur parcours scolaire au niveau de la $5^{\mathrm{e}}$ secondaire. Les taux de redoublement des garçons sont plus importants que ceux des filles, et sont nettement plus élevés dans les filières techniques et professionnelles.

Enfin, à l'intérieur des filières d'enseignement, il existe encore des choix d'options qui correspondent à des hiérarchies implicites. Ainsi, à l'intérieur de la filière générale (celle qui, en France, conduirait au bac général), on distingue des options où le programme de mathématiques et de sciences est poussé (ces options conduiraient en France au baccalauréat S), des options plus littéraires ou centrées sur les langues, d'autres centrées sur l'économie ou les sciences sociales, qui ont généralement moins bonne réputation. Un élément d'importance est à souligner : il n'existe pas de baccalauréat en Belgique. Les certificats de fin d'enseignement secondaire supérieur sont délivrés sur la base d'épreuves internes. 
sous-jacentes à cette question est que, sur différentes caractéristiques du parcours scolaire antérieur dont l'incidence sur la réussite à l'université est bien établie, garçons et filles ne se répartissent pas de façon équivalente. Autrement dit, avant de tenter d'expliquer les différences moyennes de réussite selon le sexe, il importe de vérifier que cette différence de réussite entre garçons et filles n'est pas la résultante d'autres variables du parcours scolaire antérieur - retard scolaire, filière ou choix d'options au dernier degré du secondaire. Ceci ne peut se comprendre sans avoir pris connaissance au préalable des principales caractéristiques de l'enseignement secondaire en Belgique. La structure correspond à un modèle différencié de manière relativement précoce, marqué par des filières que les élèves choisissent à partir de l'âge théorique de 14 ans, de nombreuses possibilités d'options et des taux de redoublement élevés (voir encadré).

Compte tenu de cette structure différenciée du secondaire, il n'est guère étonnant que le parcours scolaire antérieur d'un étudiant soit un déterminant important de la réussite à l'université. Ainsi, le taux de réussite de la première année universitaire varie fortement selon la filière d'enseignement secondaire suivie. Les étudiants issus du secondaire général belge (représentant plus de $90 \%$ des nouveaux entrants en 1 re année à l'université) présentent un taux de réussite supérieur à $40 \%$, tandis que ceux issus du secondaire technique de transition ont un taux de réussite inférieur à $15 \%$ et ceux sortant du secondaire technique de qualification ont un taux de réussite à peine supérieur à $5 \%^{3}$ (Ministère de la Communauté française de Belgique, 2008). Or la filière générale s'est progressivement féminisée - elle compte environ $55 \%$ de filles en dernière année du secondaire, alors que les autres filières de l'enseignement secondaire sont majoritairement masculines. Cette répartition asymétrique des filles et des garçons dans les différentes filières de l'enseignement secondaire ne permet cependant pas d'expliquer la différence de réussite à l'université entre filles et garçons puisque, à filière constante, le taux de réussite des filles reste supérieur à celui des garçons (Droesbeke, Lecrenier, Tabutin et al., 2008, p. 34 ; Ministère de la Communauté française de Belgique, 2008).

Un autre critère, l'âge à l'entrée de l'université, a également un lien avec la réussite de la première année à l'université. Ainsi, les étudiant-e-s de 18 ans et moins ("à l'heure ") ont un taux de réussite de l'ordre de $50 \%$, tandis que les étudiant-e-s de 19 ans (en retard scolaire d'un an) ont un taux de réussite de l'ordre de $30 \%$. Chez les étudiant-e-s de 20 ans et plus (en retard scolaire de plus d'un an), le taux de réussite est inférieur à $20 \%$ (Ministère de la Communauté française de Belgique, 2008). Or, selon les Indicateurs de l'enseignement (Ministère de la Communauté française de Belgique, 2011), les filles sont bien plus nombreuses que les garçons à accéder à l'université à 18 ans ou moins. Ainsi, parmi la génération des étudiants nés en 1986, les garçons issus de l'enseignement général ont été $51 \%$ à accéder à l'enseignement supérieur à 18 ans ; c'était le cas de $65 \%$ des filles. Le fait que les filles prennent moins fréquemment que les garçons du retard dans l'enseignement obligatoire ne suffit cependant pas à expliquer la différence de réussite entre étudiants et étudiantes à l'issue de la première année universitaire. En effet, les filles ont un taux de réussite supérieur à celui des garçons, à âge à l'entrée et formation secondaire suivie identiques. De plus, dans les modèles estimés par Arias Ortiz et Dehon (2008), à partir de données portant sur l'Université libre de Bruxelles ${ }^{4}$ (ULB), pour expliquer la probabilité de réussite de la première année universitaire, la prise en compte d'une variable relative au retard scolaire n'altère pas la significativité de la variable « sexe ».

Enfin, la formation en mathématiques et langues anciennes suivie dans le dernier degré du secondaire a également un lien avec la réussite à l'université. Ainsi, toutes autres choses étant égales par ailleurs, le fait d'avoir fréquenté une section du secondaire à formation mathématique forte ou avec des cours de langues anciennes semble augmenter de manière significative la probabilité de réussite de la première année à l'université (Arias Ortiz \& Dehon, 2008 ; Droesbeke, Hecquet \& Wattelar, 2001 ; Droesbeke, Lecrenier, Tabutin et al., 2008). La part des étudiants ayant suivi des cours de langues anciennes en fin de secondaire est plus élevée chez les filles que chez les garçons ; à l'inverse, la part des étudiants ayant suivi une formation forte en mathématiques en dernière année du secondaire est plus forte chez les garçons que chez les filles (Vermandele, Plaigin, Dupriez et al., 2010). Cependant, ces différences de formation entre les filles et les garçons ne permettent pas d'expliquer leur différence de réussite de la première année universitaire (Arias Ortiz \& Dehon, 2008). Dans le modèle de régression linéaire proposé par Droesbeke, Hecquet et Wattelar (2001, p. 194-202) pour expliquer le taux de réussite d'un groupe d'étudiants en fonction de leur sexe, de leur âge à l'entrée de l'université, de la section qu'ils ont fréquentée en fin de secondaire et du domaine d'études universitaires dans lequel ils se sont inscrits, la variable " sexe " exerce une influence très significative (toutes autres choses égales par ailleurs, les filles ont un taux de réussite estimé supérieur de $6 \%$ à celui des garçons). Comment dès lors expliquer 
ces différences de réussite constatées entre les filles et les garçons à l'université ? Alors que de nombreux travaux ont été consacrés à cette question pour les niveaux primaire et secondaire (Baudelot \& Establet, 1992 ; Duru-Bellat, 1989), peu de travaux ont abordé cette question au niveau des études supérieures.

Tandis que Baudelot et Establet (1992) tendent à expliquer cette meilleure réussite des filles à tous les niveaux scolaires par le fait que les filles ont des comportements et attitudes mieux adaptés aux exigences de l'école ("push factors"), DuruBellat (1989) avance une autre hypothèse : selon cette auteure, les filles "ne se coulent pas dans des stéréotypes figés, mais font des choix raisonnés et raisonnables " (Duru-Bellat, Kieffer \& Marry, 2001, p. 252), notamment par rapport au marché de l'emploi (« pull factors »). Selon Duru-Bellat, Kieffer et Marry (2001), la conjoncture du marché de l'emploi avec l'ouverture de postes de plus en plus nombreux dans le secteur tertiaire a été propice aux femmes puisqu'elle leur a permis d'accéder plus facilement à un emploi. Cependant, pour qu'elles soient engagées au sein du secteur tertiaire, il leur est de plus en plus nécessaire d'accroître leur investissement scolaire, de réussir et d'obtenir un diplôme du supérieur. Dès lors, l'investissement des filles dans le champ scolaire et leur plus grande réussite par rapport aux garçons à tous les niveaux de la scolarité s'expliqueraient notamment par la transformation du sens donné au diplôme, devenu actuellement gage d'une véritable insertion professionnelle féminine. En outre, ces auteures formulent l'hypothèse selon laquelle certaines transformations sociétales (par exemple, la création de crèches et d'écoles maternelles par l'État) et familiales (par exemple, l'adhésion à un idéal plus égalitaire dans le couple ou la réduction des familles nombreuses dans lesquelles les filles s'impliquaient souvent fortement d'un point de vue domestique) se seraient également avérées propices à l'investissement des filles dans les champs scolaire et professionnel.

Par ailleurs, les parents nourriraient aujourd'hui les mêmes attentes en matière d'éducation pour leurs filles que pour leurs garçons, voire davantage d'attentes pour leurs filles, " comme si les parents percevaient que les garçons pourront plus ou moins "se débrouiller", même avec une formation courte (souvent, du même coup, professionnelle), plus que les filles, ces dernières accédant en général à des emplois tertiaires nécessitant un bon niveau d'instruction. Certaines observations concernant le suivi de la scolarité des enfants vont dans ce sens. Ainsi, les conversations sur l'école entre parents et enfants sont un peu plus fréquentes pour les filles que pour les garçons (Terrail, 1992a) ; en particulier, les mères qui ont elles-mêmes fait des études s'avèrent très attentives à la scolarité de leur fille. Même si les mères les moins diplômées s'intéressent autant à la scolarité de leur fils qu'à celle de leur fille, la mobilisation maternelle pour la scolarité des filles apparaît sensible chez les femmes actives sans qualification (Terrail, 1992a, 1992b) » (Duru-Bellat, 1995, p. 130).

\section{Réussite selon l'origine sociale et le sexe}

La littérature sur le lien entre l'origine socioculturelle des parents et la réussite des études supérieures est des plus abondantes, tant au niveau international (Albouy \& Tavan, 2007 ; Breen, Luijks, Müller et al., 2010 ; Duru-Bellat, Kieffer \& Marry, 2001 ; Merle, 1996 ; Reay, David \& Ball, 2005 ; Shavit, Arum \& Gamaran, 2007 ; Vallet, 2010) que national (Arias Ortiz \& Dehon, 2008 ; Donni \& Pestieau, 1995 ; Droesbeke, Lecrenier, Tabutin et al., 2008). Rares sont en revanche les études qui ont abordé l'influence conjointe du sexe et de l'origine sociale, en particulier pour l'enseignement supérieur. Certains auteurs tels que Merle (1996) soulignent avec justesse que "l'étude des transformations sociales de l'Université et des CPGE [classes préparatoires aux grandes écoles] n'a en effet aucune raison de se focaliser sur la représentativité des taux d'accès selon le groupe socioprofessionnel plutôt que selon leur sexe, sauf à considérer, à tort, que les inégalités de représentation des deux sexes sont en quelque sorte mineures par rapport aux inégalités de classe " (Merle, 1996, p. 1184). En dépit de ces considérations, les analyses sont effectuées séparément pour le sexe (féminisation) et pour l'origine sociale (démocratisation), sans croiser les deux dimensions. Nous rejoignons dès lors Duru-Bellat, Kieffer et Marry (2001) lorsqu'elles soulignent la rareté d'une articulation empirique systématisée des inégalités liées à l'origine sociale et au genre sur de longues périodes ${ }^{5}$. Notre revue de la littérature se limitera donc, délibérément, aux études qui ont tenté cette articulation empirique.

De Meulemeester (2001) s'est intéressée au taux de réussite en première année des étudiants de première génération à l'Université catholique de Louvain entre 1967 et 1999. Elle observe ainsi que la réussite des filles semble, davantage que celle des garçons, liée au niveau de diplôme des parents ; que la réussite des garçons, tout comme celle des filles, dépend davantage du diplôme de la mère que de celui du père. Elle souligne enfin que le lien entre le niveau de diplôme de la mère et la réussite de la première 
année universitaire est plus fort chez les filles que chez les garçons. Alaluf, Imatouchan, Marage et alii (2003) ont de leur côté analysé les résultats d'un échantillon de 2257 étudiants s'inscrivant pour la première fois en première année à l'Université libre de Bruxelles en 2001. À l'instar de De Meulemeester (2001), ils mettent en évidence que la réussite des filles apparaît plus fortement liée au niveau de diplôme des parents que celle des garçons. Ainsi, " chez les filles, le taux de réussite est 2,5 fois plus élevé au bénéfice des enfants d'universitaires [à savoir, ici, les enfants dont les parents sont diplômés de l'université] par comparaison avec ceux dont aucun des parents ne détient un diplôme d'enseignement supérieur (ce rapport est de 1,8 chez les garçons). [...] La différence entre taux de réussite des filles et des garçons se marque par ailleurs d'autant plus que l'origine socioculturelle est élevée » (De Meulemeester, 2001, p. 100-101). En effet, lorsque les parents n'ont pas entrepris d'études supérieures, les filles et les garçons ont des taux de réussite similaires. Par contre, si les parents ont fait des études supérieures ou universitaires, les filles réussissent significativement mieux que les garçons. Les deux recherches menées en CFB au niveau des universités aboutissent donc à des résultats convergents, montrant une influence du niveau d'études des parents plus forte pour les filles que pour les garçons.

Ces résultats contrastent en revanche avec les travaux de Duru-Bellat (1989) et de Terrail (1992a, 1992b) menés en France au niveau de l'enseignement primaire et secondaire. Ceux-ci constataient, en effet, que les dissemblances de taux de réussite entre filles et garçons s'estompaient à mesure que le milieu

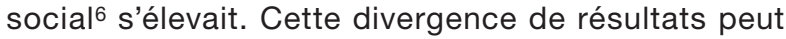
s'expliquer en partie par les différences de contexte entre les deux systèmes éducatifs et, sans doute davantage, par le fait que les travaux synthétisés par Duru-Bellat (1995) et Terrail (1992a, 1992b) portent sur l'enseignement secondaire et non sur l'enseignement supérieur7. Par ailleurs, les effets d'interaction mis en évidence par Duru-Bellat, Kieffer et Marry (2001), même s'ils sont significatifs, sont de faible ampleur. Ces auteures soulignent : « il peut exister des interactions fines entre sexe et classe. [...] II est probable que le sexe joue différemment dans certains milieux, venant tantôt creuser un avantage (ou un désavantage), tantôt l'atténuer » (Duru-Bellat, Kieffer \& Marry, 2001, p. 272).

Mais, au delà de la divergence de résultats en surface, aussi bien les résultats relatifs à l'université de De Meulemeester (2001) et d'Alaluf, Imatouchan, Marage et alii (2003) pour la CFB que ceux de Duru-Bellat
(1995) et de Terrail (1992a, 1992b) pour l'enseignement secondaire en France se rejoignent sur un phénomène de fond dont l'importance mérite d'être soulignée : il existe des influences croisées entre le genre et l'origine socioculturelle des parents. En d'autres termes, l'influence de l'origine socioculturelle des parents sur la réussite des études n'a, de nos jours, pas la même ampleur pour les filles et les garçons. Les écarts de réussite entre les garçons et les filles peuvent être davantage (dans les études françaises consacrées au secondaire) ou moins importants (dans les études belges consacrées aux études universitaires) à mesure que le diplôme des parents s'élève. Dans ses deux articles consacrés à comprendre la meilleure réussite relative des filles, Terrail (1992a, 1992b) pointe aussi une série d'effets croisés entre le genre et l'origine socioculturelle, tels qu'une ambition différentielle des filles «particulièrement élevée pour les enfants de mères actives sans qualification (ouvrières, personnes de service) dans le même type de situation scolaire (bonne ou moyenne) " (Terrail, 1992b, p. 75) ou le fait que les filles en retard scolaire restent plus ambitieuses que leurs homologues masculins. Ces travaux, même s'ils ne concernent pas les études universitaires, indiquent qu'il convient d'aborder les effets du genre et de l'origine socioculturelle de manière conjointe et d'examiner comment leurs effets se conjuguent de manière spécifique, pour le niveau d'études considéré. En la matière, l'interaction apparaît désormais comme la règle plutôt que l'exception ${ }^{8}$.

\section{MISE EN CONTEXTE : LE PAYSAGE DE L'ENSEIGNEMENT SUPÉRIEUR EN COMMUNAUTÉ FRANÇAISE DE BELGIQUE}

La Belgique francophone représente un contexte de recherche particulièrement intéressant par rapport à la question des inégalités de réussite à l'université. En effet l'accès à l'enseignement supérieur y est a priori beaucoup plus ouvert que dans la plupart des autres pays du monde. Les frais d'inscription sont modestes (l'inscription est gratuite pour les étudiants boursiers) et toutes les formes d'enseignement supérieur sont accessibles à tous les étudiants qui disposent d'un CESS (certificat d'enseignement secondaire supérieur, délivré sur la base d'examens internes à l'établissement ; il n'existe aucun examen externe obligatoire de type baccalauréat). Ainsi, à l'exception des études d'ingénieur civil, il n'y a pas en Belgique de processus de contingentement ni de sélection à l'entrée de l'enseignement supérieur ${ }^{9}$. La 
loi prévoit d'ailleurs que chacune des institutions doit accueillir tous les étudiants remplissant les conditions d'admission et qui en expriment la demande. Chaque étudiant est pratiquement assuré de trouver place dans la filière et l'institution de son choix. Alors que dans le secondaire, la concurrence entre établissements socialement hiérarchisés est vive, il n'en va pas de même dans l'enseignement supérieur. S'il existe une concurrence objective entre institutions pour attirer un nombre important d'étudiants (vu le mode de financement fondé sur le nombre d'inscriptions dans chaque institution, le budget étant constant), la formation dispensée par les différentes institutions est perçue comme étant de valeur relativement égale. L'enseignement supérieur en CFB se subdivise en trois types d'enseignement : I'université, l'enseignement supérieur hors université de type long et l'enseignement supérieur hors université de type court. On n'y trouve pas l'équivalent des grandes écoles en France. Les universités représentent le sommet de la pyramide en matière de prestige et l'enseignement universitaire est axé sur la formation théorique, en liaison avec la recherche. L'enseignement supérieur hors université de type long privilégie une formation scientifique et technique de haut niveau, plus directement orientée vers les applications. Enfin l'enseignement supérieur de type court, d'une durée minimale de trois ans, est plus directement centré sur les besoins des professions et délivre depuis la réforme de l'enseignement supérieur (réforme de Bologne) le titre de bachelier.

\section{OBJECTIFS ET MÉTHODOLOGIE}

\section{Objectifs}

La présente recherche se centre sur l'analyse du résultat (réussite ou échec) à l'issue de la première année universitaire. La première année à l'université est en effet une étape cruciale dans le parcours d'un étudiant et le résultat au terme de cette première année conditionne de manière significative la suite du cursus académique et, notamment, la probabilité pour un étudiant d'obtenir un diplôme universitaire (Droesbeke, Lecrenier, Tabutin et al., 2008). Les objectifs poursuivis dans cette recherche sont les suivants:

- évaluer la relation entre la réussite en première année à l'université et le sexe, tout en contrôlant le niveau d'études des parents et la trajectoire scolaire antérieure des étudiants (voir la sous-partie "Réussite selon le sexe ") ;
- vérifier s'il existe, dans la relation qui les lie à la réussite de la première année universitaire, une interaction entre le sexe de l'étudiant et le niveau d'études de ses parents. Plus précisément, il s'agit de vérifier si la relation entre la réussite en première année et le sexe se marque de la même façon dans les différents milieux sociaux (voir la sous-partie "Réussite selon le sexe et le niveau d'études des parents ") ; et de vérifier d'autre part si le diplôme des parents est lié de la même façon à la réussite en première année selon le sexe (voir la sous-partie «Réussite et niveau d'études des parents selon le sexe $»$ ).

Nous avons également envisagé, dans nos analyses, la possibilité que les écarts de réussite entre garçons et filles et les interactions entre le sexe et le niveau de diplôme des parents se modulent selon le domaine d'études (sciences humaines et sociales, sciences exactes, sciences de la santé). L'interprétation de ces résultats s'est toutefois révélée ardue, appelant des développements importants et spécifiques, qui ne pouvaient trouver place dans l'espace du présent article. Nous avons donc décidé de ne pas inclure ces résultats. Nous y ferons toutefois écho brièvement lors de la présentation des résultats et de la discussion ${ }^{10}$.

\section{La base de données}

L'étude utilise une base de données de la population des étudiants inscrits pour la première fois en première année en 2001-2002 dans deux universités de la CFB, à savoir l'Université catholique de Louvain (50,9\%) et l'Université libre de Bruxelles (49,1\%), qui sont les deux institutions qui comptent le plus d'étudiants. L'échantillon se compose de 2927 étudiants ${ }^{11 .}$

\section{Les variables}

Les variables prises en compte pour tenter d'expliquer la probabilité de réussite de la première année à l'université peuvent être regroupées en trois catégories différentes : les caractéristiques individuelles, ici le sexe de l'étudiant; les caractéristiques du parcours scolaire antérieur et l'environnement socioculturel. Pour le parcours antérieur, nous distinguons :

- la variable indiquant si l'étudiant a obtenu son certificat d'enseignement secondaire (ci-après CESS) " à l'heure " (à 18 ans ou moins) ou en retard (à 19 ans ou plus) ;

- le nombre d'heures de mathématiques hebdomadaires suivies dans le dernier degré du secondaire : pas 
plus de trois heures de mathématiques, quatre ou cinq heures, six heures de mathématiques ou plus. Pour certaines analyses, les deux premières catégories sont regroupées pour obtenir un effectif suffisant ;

- la variable indiquant si l'étudiant suivait ou non, en dernier degré du secondaire, des cours de langues anciennes.

Pour l'environnement socioculturel12, les variables sont :

- le niveau de diplôme du père selon trois modalités (pas de diplôme du supérieur, diplôme du supérieur hors universités, SHU par la suite, diplôme universitaire), les deux premières modalités seront souvent regroupées en une seule (pas de diplôme universitaire) pour faciliter la lecture et l'interprétation des résultats ;

- le niveau de diplôme de la mère : mêmes modalités que pour la variable précédente.

Pour certaines analyses, le niveau de diplôme des deux parents sera envisagé conjointement au travers d'une variable appelée " Niveau d'études des parents ", définie soit en trois modalités (aucun parent diplômé du supérieur, au moins un parent diplômé de l'enseignement supérieur, mais aucun parent diplômé de l'université et au moins un parent diplômé de l'université), soit sous une forme binaire (parents diplômés de l'université ou non).

\section{La régression logistique binaire}

La régression logistique est un cas particulier du modèle linéaire. Dans le cas d'une régression logistique binaire, la variable dépendante est dichotomique et l'objectif est de prévoir la probabilité qu'un individu soit classé dans l'une ou l'autre catégorie (ici réussite ou échec) à partir d'une ou de plusieurs variables (par exemple le sexe ou l'origine socioculturelle). Divers indices permettent de mesurer la qualité d'ajustement du modèle aux données observées et ainsi, d'une certaine façon, le pouvoir explicatif du modèle. Dans le cas présent, il s'agit du coefficient de détermination $R^{2}$ de Nagelkerke (pseudo- $R^{2}$ ) dont la valeur est comprise entre 0 et 1 . Ce coefficient quantifie dans quelle mesure le modèle incluant les variables explicatives s'ajuste mieux aux données que le modèle sans variables explicatives : un $\mathrm{R}^{2}$ proche de 0 signifie que l'apport des variables explicatives est extrêmement faible; plus $R^{2}$ est proche de 1 , plus les variables explicatives permettent un bon ajustement aux données.

L'interprétation des coefficients de régression se fait le plus souvent à travers l'analyse des odds ratio (OR) ou rapports de chances. On définit ainsi, par exemple, la " chance " (odd) de réussite à l'université d'un étudiant comme étant le rapport entre sa probabilité de réussite et sa probabilité de non-réussite (échec). Supposons par exemple que la première variable explicative soit le sexe de l'étudiant. On peut alors définir le rapport de chances (odds ratio ou OR) associé à la variable comme étant le rapport entre la "chance » de réussite à l'université d'une fille et celle d'un garçon, toutes autres choses étant égales par ailleurs. Un signe positif pour le coefficient de régression associé à la variable signifie alors qu'OR est strictement supérieur à 1, c'est-à-dire que la " chance " de réussite d'une fille est supérieure à celle d'un garçon, toutes autres choses étant égales par ailleurs ; un signe négatif du coefficient de régression signifie au contraire qu'OR est strictement inférieur à 1 et donc que la "chance » de réussite à l'université d'une fille est inférieure à celle d'un garçon. Dans nos analyses, un OR d'une valeur de 2, par exemple, sera interprété en affirmant que la chance de réussite à l'université d'une fille est deux fois supérieure à celle d'un garçon ayant les mêmes caractéristiques par ailleurs.

\section{LES RÉSULTATS}

\section{Réussite selon le sexe}

Avant d'évaluer la relation entre le résultat au terme de la première année universitaire (réussite ou échec) et le sexe par un modèle de régression logistique, nous avons effectué quelques analyses descriptives du taux de réussite en première année à l'université (voir le tableau 1). De nettes différences selon le sexe apparaissent : le taux de réussite en première année à l'université est de 32,4\% parmi les garçons et 43,1\% parmi les filles. Quel que soit le secteur d'études, les filles réussissent plus fréquemment leur première année universitaire que les garçons. Cependant, si le sexe est un déterminant très significatif de la réussite (même lorsqu'on contrôle les autres variables) dans le secteur des sciences humaines et sociales et celui des sciences (hors sciences de l'ingénieur), il n'est plus statistiquement significatif dans le secteur des sciences de la santé13. Même si notre échantillon ne porte que sur deux institutions universitaires, les taux de réussite observés ne s'écartent qu'assez faiblement 
des taux de réussite dans la population de référence. En effet, selon les données du CREF (2003) qui portent sur l'ensemble des institutions universitaires de la CFB, pour l'année académique 2001-2002, le taux de réussite des étudiants de première génération est de $34,3 \%$ pour les garçons et $42,7 \%$ pour les filles. Au delà de la différence moyenne de réussite selon le sexe, il faut noter qu'à situation équivalente (retard ou non dans l'obtention du certificat de fin d'études secondaires, formation en mathématiques ou en langues anciennes, niveau d'études du père ou de la mère), les filles réussissent systématiquement mieux que les garçons.

Évoquons maintenant les régressions logistiques. Dans le premier modèle, seul le sexe a été introduit comme variable indépendante. La colonne "modèle 1 " du tableau 2 indique que la probabilité de réussite de la première année universitaire diffère significativement selon le sexe (odds ratio significatif).

Tableau 1. Taux de réussite observés dans l'échantillon

\begin{tabular}{|c|c|c|c|c|c|c|c|c|c|}
\hline & \multicolumn{3}{|c|}{ Tous } & \multicolumn{3}{|c|}{ Garçons } & \multicolumn{3}{|c|}{ Filles } \\
\hline & Effectif & $\begin{array}{l}\text { Taux de } \\
\text { réussite }\end{array}$ & \begin{tabular}{c|}
$\mathrm{V}$ \\
de Cramer
\end{tabular} & Effectif & $\begin{array}{l}\text { Taux de } \\
\text { réussite }\end{array}$ & $\begin{array}{c}\text { V } \\
\text { de Cramer }\end{array}$ & Effectif & $\begin{array}{l}\text { Taux de } \\
\text { réussite }\end{array}$ & $\begin{array}{c}\text { V } \\
\text { de Cramer }\end{array}$ \\
\hline Garçons & 1260 & $32,4 \%$ & \multirow{2}{*}{0,109} & & & & & & \\
\hline Filles & 1667 & $43,1 \%$ & & & & & & & \\
\hline $\begin{array}{l}\text { CESS } \\
\text { à l'heure }\end{array}$ & 2741 & $40,6 \%$ & \multirow{2}{*}{0,169} & 1147 & $35,3 \%$ & \multirow{2}{*}{0,199} & 1594 & $44,4 \%$ & \multirow{2}{*}{0,127} \\
\hline $\begin{array}{l}\text { CESS } \\
\text { en retard }\end{array}$ & 186 & $7,0 \%$ & & 113 & $2,7 \%$ & & 73 & $13,7 \%$ & \\
\hline Maths $0-3 \mathrm{~h}$ & 184 & $28,3 \%$ & \multirow{3}{*}{0,160} & 70 & $30,0 \%$ & \multirow{3}{*}{0,210} & 114 & $27,2 \%$ & \multirow{3}{*}{0,171} \\
\hline Maths 4-5h & 1414 & $31,8 \%$ & & 499 & $20,6 \%$ & & 915 & $37,9 \%$ & \\
\hline Maths $6 \mathrm{~h}$ et + & 1329 & $47,0 \%$ & & 691 & $41,1 \%$ & & 638 & $53,3 \%$ & \\
\hline $\begin{array}{l}\text { Pas de } \\
\text { langues } \\
\text { anciennes }\end{array}$ & 2018 & $31,3 \%$ & \multirow[t]{2}{*}{0,219} & 964 & $27,2 \%$ & \multirow[t]{2}{*}{0,201} & 1054 & $35,1 \%$ & \multirow[t]{2}{*}{0,211} \\
\hline $\begin{array}{l}\text { Langues } \\
\text { anciennes }\end{array}$ & 909 & $54,3 \%$ & & 296 & $49,3 \%$ & & 613 & $56,8 \%$ & \\
\hline $\begin{array}{l}\text { Père non } \\
\text { diplômé du } \\
\text { supérieur }\end{array}$ & 1031 & $29,1 \%$ & \multirow{3}{*}{0,148} & 404 & $23,8 \%$ & \multirow{3}{*}{0,139} & 627 & $32,5 \%$ & \multirow{3}{*}{0,171} \\
\hline Père SHU & 718 & $40,3 \%$ & & 295 & $31,9 \%$ & & 423 & $46,1 \%$ & \\
\hline $\begin{array}{l}\text { Père diplômé } \\
\text { de l'université }\end{array}$ & 1178 & $45,6 \%$ & & 561 & $38,9 \%$ & & 617 & $51,7 \%$ & \\
\hline $\begin{array}{l}\text { Mère non } \\
\text { diplômée du } \\
\text { supérieur }\end{array}$ & 1076 & $28,5 \%$ & \multirow{3}{*}{0,164} & 438 & $23,1 \%$ & \multirow{3}{*}{0,150} & 638 & $32,3 \%$ & \multirow{3}{*}{0,185} \\
\hline Mère SHU & 1161 & $41,8 \%$ & & 498 & $35,5 \%$ & & 663 & $46,5 \%$ & \\
\hline $\begin{array}{l}\text { Mère } \\
\text { diplômée de } \\
\text { l'université }\end{array}$ & 690 & $48,4 \%$ & & 324 & $40,1 \%$ & & 366 & $55,7 \%$ & \\
\hline Total & 2927 & $38,5 \%$ & & 1260 & $32,4 \%$ & & 1667 & $43,1 \%$ & \\
\hline
\end{tabular}

Note : le V de Cramer $^{14}$ (compris entre 0 et 1) mesure l'intensité de l'association entre deux variables nominales. Plus $V$ est proche de 1 , plus la relation entre les deux variables est forte ; plus $V$ est proche de 0 , plus on s'approche de la situation d'indépendance entre les deux variables étudiées. Pour alléger le tableau, nous n'avons pas inclus les seuils de significativité. Tous les $V$ de Cramer sont significatifs à au moins 0,0001 . 
Tableau 2. Résultats des estimations des modèles logistiques pour la réussite selon le sexe (la taille de l'échantillon est de 2927 élèves)

\begin{tabular}{|c|c|c|c|c|c|c|c|c|c|c|}
\hline \multirow{2}{*}{ Références } & \multirow[b]{2}{*}{ Modalités } & \multicolumn{3}{|c|}{ Modèle 1} & \multicolumn{3}{|c|}{ Modèle 2} & \multicolumn{3}{|c|}{ Modèle 3} \\
\hline & & Coeff. & Sig. & OR & Coeff. & Sig. & OR & Coeff. & Sig. & OR \\
\hline Garçon & Fille & $0,457^{\star \star \star}$ & 0,000 & 1,58 & $0,514^{* * *}$ & 0,000 & 1,67 & $0,499^{* * *}$ & 0,000 & 1,65 \\
\hline $\begin{array}{l}\text { CESS } \\
\text { à l'heure }\end{array}$ & Retard CESS & & & & & & & $-1,654^{\star \star *}$ & 0,000 & 0,19 \\
\hline Maths $0-5 \mathrm{~h}$ & Maths $6 \mathrm{~h}$ et + & & & & & & & $0,726^{\star \star \star}$ & 0,000 & 2,07 \\
\hline $\begin{array}{l}\text { Pas de } \\
\text { langues } \\
\text { anciennes }\end{array}$ & $\begin{array}{l}\text { Langues } \\
\text { anciennes }\end{array}$ & & & & & & & $0,870^{\star \star \star}$ & 0,000 & 2,39 \\
\hline $\begin{array}{l}\text { Pas de } \\
\text { parent } \\
\text { diplômé de } \\
\text { l'université }\end{array}$ & $\begin{array}{l}\text { Au moins un } \\
\text { parent diplômé de } \\
\text { l'université }\end{array}$ & & & & $0,569^{\star * \star}$ & 0,000 & 1,77 & $0,439^{\star \star \star}$ & 0,000 & 1,55 \\
\hline \multicolumn{2}{|c|}{ Coefficient de détermination } & \multicolumn{3}{|c|}{$R^{2}=0,016$} & \multicolumn{3}{|c|}{$R^{2}=0,041$} & \multicolumn{3}{|c|}{$R^{2}=0,151$} \\
\hline
\end{tabular}

Note : « sig. » est la significativité des résultats : ${ }^{*}$ : résultat significatif à 0,$05 ;{ }^{* *}$ : résultat significatif à 0,$01 ;{ }^{* * *}$ : résultat significatif à $0,001$.

En outre, l'odds ratio étant supérieur à 1 (la modalité " garçon » étant la référence), nous pouvons en conclure que la chance pour une fille de réussir sa première année à l'université est en moyenne 1,6 fois plus élevée que celle d'un garçon. Cet odds ratio varie selon les domaines d'études. Ainsi, en sciences, où les filles sont proportionnellement moins nombreuses, leur chance de réussite est nettement plus élevée que celle des garçons (2,3 fois plus élevée), sans doute en raison d'un phénomène de sursélection. En revanche, en sciences humaines et sociales, ainsi qu'en sciences de la santé, le différentiel de réussite est moins marqué (respectivement 1,7 et 1,4 fois plus de chances de réussir pour une fille).

Dans un deuxième temps, nous avons ajouté au modèle (colonne « modèle 2 » du tableau 2 ) la variable "parents diplômés de l'université ou non ", variable qui s'avère significative : toutes choses étant égales par ailleurs, la chance de réussir la première année à l'université est 1,8 fois plus élevée pour un jeune dont au moins un des parents est diplômé de l'université par rapport à un jeune dont aucun des parents n'est diplômé de l'université15. En outre, l'introduction de cette nouvelle variable permet d'améliorer quelque peu le pouvoir explicatif du modèle $\left(R^{2}\right.$ passe de 0,016 à 0,041 ) et ne modifie pas l'influence de la variable sexe (OR toujours significatif et du même ordre de grandeur que dans le premier modèle) : avec les mêmes caractéristiques de diplôme des parents, la chance de réussir la première année à l'université reste 1,6 fois plus élevée pour une fille que pour un garçon.
Enfin, pour le modèle 3 , trois caractéristiques du parcours scolaire antérieur ont été ajoutées comme variables explicatives. II en résulte une forte augmentation du pouvoir explicatif du modèle $\left(R^{2}\right.$ passe de 0,041 à 0,151$)$. Chacune de ces trois caractéristiques ressort comme étant fortement significative. Toutes autres choses étant égales par ailleurs, la chance de réussir la première année universitaire est 5,2 (inverse de 0,191) fois plus élevée si l'étudiant a obtenu son certificat de fin d'études secondaires "à l'heure ", 2,1 fois plus élevée pour un étudiant sortant de " maths fortes " et 2,4 fois plus élevée pour un étudiant issu d'une section du secondaire avec des langues anciennes. Mais, même en contrôlant ces caractéristiques du parcours scolaire, la probabilité de réussite reste significativement différente pour les filles et les garçons : avec la même situation en ce qui concerne l'âge d'obtention du certificat de fin d'études secondaires, la même formation en mathématiques et en langues anciennes, et le même niveau d'études des parents, les filles ont 1,6 fois plus de chances de réussir leur première année à l'université que les garçons. Comme nous l'avions déjà souligné dans l'état de la question, les différences de réussite observées entre les filles et les garçons ne sont donc pas seulement dues à des différences de parcours au sein du secondaire. Observons également que l'introduction dans le modèle des caractéristiques du parcours scolaire antérieur ne modifie pratiquement pas l'influence du niveau d'études des parents sur la probabilité de réussite : toutes autres choses étant égales par ailleurs, les étudiants dont le père et/ou 
Tableau 3. Taux de réussite des garçons et des filles selon le niveau d'études des parents

\begin{tabular}{|l|c|c|c|}
\cline { 2 - 4 } \multicolumn{1}{c|}{} & $\begin{array}{c}\text { Aucun parent diplômé } \\
\text { du supérieur }\end{array}$ & $\begin{array}{c}\text { Au moins un parent diplômé } \\
\text { du supérieur, mais aucun } \\
\text { de l'université }\end{array}$ & $\begin{array}{c}\text { Au moins un parent } \\
\text { diplômé de l'université }\end{array}$ \\
\hline Garçons & $20,6 \%$ & $30,8 \%$ & $38,2 \%$ \\
\hline Filles & $28,8 \%$ & $43,4 \%$ & $51,4 \%$ \\
\hline OR & 1,56 & 1,72 & 1,71 \\
\hline
\end{tabular}

Tableau 4. Résultats des estimations des modèles logistiques selon le niveau d'études des parents

\begin{tabular}{|c|c|c|c|c|c|c|c|c|c|c|}
\hline \multirow[b]{2}{*}{ Références } & \multirow[b]{2}{*}{ Modalités } & \multicolumn{3}{|c|}{$\begin{array}{c}\text { Aucun parent diplômé du } \\
\text { supérieur }\end{array}$} & \multicolumn{3}{|c|}{$\begin{array}{c}\text { Au moins un parent diplômé } \\
\text { du supérieur mais aucun } \\
\text { de l'université }\end{array}$} & \multicolumn{3}{|c|}{$\begin{array}{l}\text { Au moins un parent } \\
\text { diplômé de l'université }\end{array}$} \\
\hline & & Coeff. & Sig. & OR & Coeff. & Sig. & OR & Coeff. & Sig. & OR \\
\hline Garçon & Fille & $0,412^{*}$ & 0,034 & 1,51 & $0,514^{\star \star *}$ & 0,001 & 1,67 & $0,544^{\star \star \star}$ & 0,000 & 1,72 \\
\hline $\begin{array}{l}\text { CESS } \\
\text { à l'heure }\end{array}$ & \begin{tabular}{|l|} 
Retard \\
CESS \\
\end{tabular} & $-1,225^{\star \star}$ & 0,006 & 0,29 & $-2,971^{\star *}$ & 0,003 & 0,05 & $-1,455^{\star \star \star}$ & 0,001 & 0,23 \\
\hline Maths 0-5h & $\begin{array}{l}\text { Maths 6h } \\
\text { et }+\end{array}$ & $0,615^{\star \star \star}$ & 0,001 & 1,85 & $0,588^{\star \star \star}$ & 0,000 & 1,80 & $0,853^{\star * *}$ & 0,000 & 2,35 \\
\hline $\begin{array}{l}\text { Pas de } \\
\text { langues } \\
\text { anciennes }\end{array}$ & $\begin{array}{l}\text { Langues } \\
\text { anciennes }\end{array}$ & $0,536^{\star *}$ & 0,007 & 1,71 & $0,751^{\star * *}$ & 0,000 & 2,12 & $1,053^{\star * \star}$ & 0,000 & 2,87 \\
\hline \multicolumn{2}{|c|}{$\begin{array}{l}\text { Coefficient de } \\
\text { détermination }\end{array}$} & \multicolumn{3}{|c|}{$R^{2}=0,081$} & \multicolumn{3}{|c|}{$R^{2}=0,128$} & \multicolumn{3}{|c|}{$R^{2}=0,158$} \\
\hline
\end{tabular}

Note : « sig. » est la significativité des résultats : ${ }^{*}$ : résultat significatif à 0,$05 ;{ }^{* \star}$ : résultat significatif à 0,$01 ;{ }^{* \star *}:$ résultat significatif à $0,001$.

la mère est diplômé de l'université ont toujours près de 1,5 fois plus de chances de réussir leur première année à l'université que ceux dont les parents ne sont pas diplômés de l'université.

\section{Réussite selon le sexe et le niveau d'études des parents}

Notre deuxième objectif était de vérifier s'il existe, dans la relation qui les lie à la réussite de la première année universitaire, une interaction entre le sexe de l'étudiant et le niveau d'études de ses parents. Pour atteindre cet objectif, nous procédons de deux manières : tout d'abord, nous cherchons, au sein de cette sous-partie, à vérifier si la relation entre la réussite en première année et le sexe se marque de la même façon selon que les parents ont un diplôme plus ou moins élevé ; ensuite, nous prenons le problème dans l'autre sens en analysant les différences d'impact du niveau d'études des parents sur la réussite en première année selon le sexe. Avant de présenter les modèles de régression logistique, nous avons effectué quelques analyses descriptives des taux de réussite des filles et garçons en première année selon le niveau d'études des parents. À la lecture du tableau 3 , de nettes différences entre étudiants masculins et féminins apparaissent selon le diplôme des parents. Ainsi la différence de réussite entre les garçons et les filles est plus marquée chez les jeunes dont au moins un parent est diplômé du supérieur ou de l'université que chez les jeunes dont aucun parent n'est diplômé du supérieur ${ }^{16}$.

Nous avons ensuite fait tourner trois modèles de régression logistique, un modèle par niveau d'études des parents (voir le tableau 4). La différence de réussite entre les garçons et les filles est d'autant plus marquée que le niveau d'études des parents est élevé, et cela même si l'on contrôle les caractéristiques du 
Tableau 5a. Résultats des estimations des modèles logistiques chez les garçons pour la réussite et selon le diplôme universitaire des parents

\begin{tabular}{|c|c|c|c|c|c|c|c|}
\hline & & & odèle 1 & & & Ddèle 2 & \\
\hline Références & Modalités & Coeff. & Sig. & OR & Coeff. & Sig. & OR \\
\hline CESS à l'heure & Retard CESS & & & & $-2,463^{\star \star \star}$ & 0,000 & 0,08 \\
\hline Maths $0-5 \mathrm{~h}$ & Maths $6 \mathrm{~h}$ et + & & & & $0,831^{* \star \star}$ & 0,000 & 2,30 \\
\hline $\begin{array}{l}\text { Pas de langues } \\
\text { anciennes }\end{array}$ & Langues anciennes & & & & $0,926^{\star \star \star}$ & 0,000 & 2,52 \\
\hline $\begin{array}{l}\text { Pas de parent } \\
\text { diplômé de } \\
\text { l'université }\end{array}$ & $\begin{array}{l}\text { Au moins un parent } \\
\text { diplômé de l'université }\end{array}$ & $0,542^{\star \star *}$ & 0,000 & 1,72 & $0,383^{\star \star}$ & 0,003 & 1,47 \\
\hline \multicolumn{2}{|c|}{ Coefficient de détermination } & \multicolumn{3}{|c|}{$R^{2}=0,022$} & \multicolumn{3}{|c|}{$\mathrm{R}^{2}=0,165$} \\
\hline
\end{tabular}

Note : « sig. " est la significativité des résultats : * : résultat significatif à 0,$05 ;{ }^{* *}$ : résultat significatif à 0,$01 ;{ }^{* \star *}:$ résultat significatif à 0,001 .

Tableau 5b. Résultats des estimations des modèles logistiques chez les filles pour la réussite et selon le diplôme universitaire des parents

\begin{tabular}{|c|c|c|c|c|c|c|c|}
\hline & & & odèle & & & odèle 2 & \\
\hline Références & Modalités & Coeff. & Sig. & OR & Coeff. & Sig. & OR \\
\hline CESS à l'heure & Retard CESS & & & & $-1,140^{\star \star *}$ & 0,001 & 0,32 \\
\hline Maths $0-5 \mathrm{~h}$ & Maths $6 \mathrm{~h}$ et + & & & & $0,656^{\star \star \star}$ & 0,000 & 1,93 \\
\hline $\begin{array}{l}\text { Pas de langues } \\
\text { anciennes }\end{array}$ & Langues anciennes & & & & $0,844^{* * *}$ & 0,000 & 2,32 \\
\hline $\begin{array}{l}\text { Pas de parent } \\
\text { diplômé de } \\
\text { l'université }\end{array}$ & $\begin{array}{l}\text { Au moins un } \\
\text { parent diplômé de } \\
\text { l'université }\end{array}$ & $0,588^{\star \star \star}$ & 0,000 & 1,80 & $0,475^{\star \star \star}$ & 0,000 & 1,61 \\
\hline Coefficient de d & ermination & & $=0,02$ & & & $=0,12$ & \\
\hline
\end{tabular}

Note : « sig. " est la significativité des résultats : ${ }^{\star}$ : résultat significatif à 0,$05 ;{ }^{\star \star}$ : résultat significatif à 0,$01 ;{ }^{* \star \star}:$ résultat significatif à $0,001$.

parcours scolaire antérieur. En effet, toutes autres choses étant égales par ailleurs, la chance de réussite de la première année universitaire des filles est 1,5 fois plus élevée que celle des garçons lorsqu'aucun parent n'est diplômé de l'enseignement supérieur, 1,7 fois plus élevée lorsqu'au moins un parent est diplômé de l'enseignement supérieur mais que ni le père, ni la mère ne sont diplômés de l'université, 1,7 fois plus élevée lorsqu'au moins un parent est diplômé de l'université. Observons également que le pouvoir explicatif du modèle prenant en compte le sexe et les caractéristiques du parcours scolaire antérieur s'accroît avec le niveau d'études des parents : le $\mathrm{R}^{2}$ de Nagelkerke passe de 0,081 lorsqu'aucun parent n'est diplômé de l'enseignement supérieur à 0,158 lorsqu'au moins un parent est diplômé de l'université.

\section{Réussite et niveau d'études des parents selon le sexe}

Le niveau d'études des parents a-t-il une influence similaire sur la réussite de la première année universitaire chez les filles et chez les garçons ? Pour tenter de répondre à cette question, nous avons comparé les estimations des modèles logistiques réalisées séparément pour les filles et pour les garçons. Chez les garçons comme chez les filles, le fait d'avoir au moins un parent diplômé de l'université a un impact positif statistiquement significatif sur la réussite (cf. les tableaux 5a et 5b). Non seulement cet impact reste statistiquement significatif lorsqu'on contrôle les caractéristiques du parcours scolaire antérieur, mais il s'avère également plus marqué pour les filles que pour les garçons : à parcours scolaire identique, le rapport de chances de réussite d'un jeune ayant au 
Tableau 6a. Résultats des estimations des modèles logistiques chez les garçons pour la réussite et selon le diplôme universitaire du père et de la mère

\begin{tabular}{|c|c|c|c|c|c|c|c|c|c|c|c|c|c|}
\hline \multirow[b]{2}{*}{ Références } & \multirow[b]{2}{*}{ Modalités } & \multicolumn{3}{|c|}{ Modèle 1} & \multicolumn{3}{|c|}{ Modèle 2} & \multicolumn{3}{|c|}{ Modèle 3} & \multicolumn{3}{|c|}{ Modèle 4} \\
\hline & & Coeff. & Sig. & OR & Coeff. & Sig. & OR & Coeff. & Sig. & OR & Coeff. & Sig. & OR \\
\hline $\begin{array}{l}\text { CESS } \\
\text { à l'heure }\end{array}$ & $\begin{array}{l}\text { Retard } \\
\text { CESS }\end{array}$ & & & & & & & & & & $-2,469^{* * *}$ & 0,000 & 0,08 \\
\hline Maths $0-5 \mathrm{~h}$ & $\begin{array}{l}\text { Maths 6h } \\
\text { et }+\end{array}$ & & & & & & & & & & $0,825^{\star \star *}$ & 0,000 & 2,28 \\
\hline $\begin{array}{l}\text { Pas de } \\
\text { langues } \\
\text { anciennes }\end{array}$ & $\begin{array}{l}\text { Langues } \\
\text { anciennes }\end{array}$ & & & & & & & & & & $0,922^{\star \star \star}$ & 0,000 & 2,51 \\
\hline $\begin{array}{l}\text { Père non } \\
\text { diplômé de } \\
\text { l'université }\end{array}$ & $\begin{array}{l}\text { Père } \\
\text { diplômé } \\
\text { de } \\
\text { l'université }\end{array}$ & $0,532^{\star \star \star}$ & 0,000 & 1,70 & & & & $0,438^{* * *}$ & 0,001 & 1,55 & $0,354^{*}$ & 0,011 & 1,42 \\
\hline $\begin{array}{l}\text { Mère non } \\
\text { diplômée } \\
\text { de } \\
\text { l'université }\end{array}$ & $\begin{array}{l}\text { Mère } \\
\text { diplômée } \\
\text { de } \\
\text { l'université }\end{array}$ & & & & $0,461^{\star \star \star}$ & 0,001 & 1,59 & 0,270 & 0,065 & 1,31 & 0,110 & 0,473 & 1,12 \\
\hline \multicolumn{2}{|c|}{$\begin{array}{l}\text { Coefficient de } \\
\text { détermination }\end{array}$} & \multicolumn{3}{|c|}{$R^{2}=0,021$} & \multicolumn{3}{|c|}{$R^{2}=0,013$} & \multicolumn{3}{|c|}{$\mathrm{R}^{2}=0,025$} & \multicolumn{3}{|c|}{$R^{2}=0,166$} \\
\hline
\end{tabular}

Note : « sig. » est la significativité des résultats : ${ }^{*}$ : résultat significatif à 0,$05 ;{ }^{* \star}$ : résultat significatif à 0,$01 ;{ }^{* \star *}:$ résultat significatif à $0,001$.

Tableau 6b. Résultats des estimations des modèles logistiques chez les filles pour la réussite et selon le diplôme universitaire du père et de la mère

\begin{tabular}{|c|c|c|c|c|c|c|c|c|c|c|c|c|c|}
\hline \multirow[b]{2}{*}{ Références } & \multirow[b]{2}{*}{ Modalités } & \multicolumn{3}{|c|}{ Modèle 1} & \multicolumn{3}{|c|}{ Modèle 2} & \multicolumn{3}{|c|}{ Modèle 3} & \multicolumn{3}{|c|}{ Modèle 4} \\
\hline & & Coeff. & Sig. & OR & Coeff. & Sig. & OR & Coeff. & Sig. & OR & Coeff. & Sig. & OR \\
\hline $\begin{array}{l}\text { CESS } \\
\text { à l'heure }\end{array}$ & $\begin{array}{l}\text { Retard } \\
\text { CESS }\end{array}$ & & & & & & & & & & $-1,151^{\star \star \star}$ & 0,001 & 0,32 \\
\hline $\begin{array}{l}\text { Maths } \\
0-5 \mathrm{~h}\end{array}$ & $\begin{array}{l}\text { Maths 6h } \\
\text { et }+\end{array}$ & & & & & & & & & & $0,640^{\star \star *}$ & 0,000 & 1,90 \\
\hline $\begin{array}{l}\text { Pas de } \\
\text { langues } \\
\text { anciennes }\end{array}$ & $\begin{array}{l}\text { Langues } \\
\text { anciennes }\end{array}$ & & & & & & & & & & $0,837^{\star \star \star}$ & 0,000 & 2,31 \\
\hline $\begin{array}{l}\text { Père non } \\
\text { diplômé de } \\
\text { l'université }\end{array}$ & \begin{tabular}{|l|} 
Père \\
diplômé \\
de \\
l'université
\end{tabular} & $0,558^{\star \star \star}$ & 0,000 & 1,75 & & & & $0,385^{\star \star \star}$ & 0,001 & 1,47 & $0,325^{\star *}$ & 0,006 & 1,38 \\
\hline $\begin{array}{l}\text { Mère non } \\
\text { diplômée } \\
\text { de } \\
\text { l'université }\end{array}$ & \begin{tabular}{|l|} 
Mère \\
diplômée \\
de \\
l'université
\end{tabular} & & & & $0,657^{* \star *}$ & 0,000 & 1,93 & $0,461^{* \star *}$ & 0,001 & 1,59 & $0,355^{* *}$ & 0,010 & 1,43 \\
\hline \multicolumn{2}{|c|}{$\begin{array}{l}\text { Coefficient de } \\
\text { détermination }\end{array}$} & \multicolumn{3}{|c|}{$\mathrm{R}^{2}=0,024$} & \multicolumn{3}{|c|}{$\mathrm{R}^{2}=0,024$} & \multicolumn{3}{|c|}{$R^{2}=0,033$} & \multicolumn{3}{|c|}{$\mathrm{R}^{2}=0,124$} \\
\hline
\end{tabular}

Note : « sig. " est la significativité des résultats : ${ }^{\star}$ : résultat significatif à 0,$05 ;{ }^{\star \star}$ : résultat significatif à 0,$01 ;{ }^{* \star \star}:$ résultat significatif à $0,001$. 
moins un parent diplômé de l'université par rapport à un condisciple dont aucun parent n'est diplômé de l'enseignement supérieur s'élève à 1,5 chez les garçons et à 1,6 chez les filles.

Par ailleurs, l'influence respective du niveau d'études du père et de la mère sur la réussite ne semble pas s'exercer de la même façon pour les deux sexes, comme le montre l'estimation des modèles logistiques dans lesquels on distingue le niveau de diplôme du père de celui de la mère (cf. les tableaux $6 a$ et $6 b$ ). Le fait d'avoir un père diplômé de l'université a un impact positif statistiquement significatif sur la probabilité de réussite chez les garçons et chez les filles. Le fait d'avoir une mère diplômée de l'université favorise également positivement la réussite. Cependant si, chez les filles, l'impact du niveau d'études de la mère reste statistiquement significatif lorsqu'on contrôle le niveau d'études du père et les variables caractérisant le parcours scolaire antérieur, ce n'est pas le cas chez les garçons.

En outre, les trois variables prises en compte pour caractériser le parcours scolaire antérieur semblent avoir un pouvoir explicatif sur la réussite plus élevé chez les garçons que chez les filles. En effet, l'introduction de ces variables dans le modèle permet une plus grande augmentation du $\mathrm{R}^{2}$ de Nagelkerke et donne ainsi un pouvoir explicatif plus élevé au modèle logistique chez les garçons, quelle que soit la variable considérée pour prendre en compte le niveau d'études du père et/ou de la mère (cf. les tableaux $5 a, 5 b, 6 a$ et $6 b)$.

\section{DISCUSSION}

Le principal objectif de cet article était d'analyser les différences de réussite entre filles et garçons en première année universitaire, sous contrôle des variables socioculturelles et des parcours scolaires antérieurs. Cet objectif a été approché à travers trois analyses successives. Comme nous l'avions mis en exergue dans l'état de la question, les différences de réussite à l'université entre les filles et les garçons ne sont pas la simple résultante d'un parcours différencié au sein du secondaire. Avec la même situation en ce qui concerne l'âge d'obtention du certificat de fin d'études secondaires, la même formation en mathématiques et en langues anciennes, et à même niveau d'études des parents, les filles ont toujours 1,6 fois plus de chances de réussir leur première année à l'université que les garçons.
Nous avons ensuite voulu vérifier si la relation entre la réussite en première année et le sexe se marquait de la même façon selon le niveau d'études des parents. Comme Duru-Bellat (1995) et Terrail (1992b) l'avaient constaté pour l'enseignement secondaire en France, les différences de réussite entre les garçons et les filles s'estompent-elles lorsque le milieu social s'élève ou au contraire celles-ci s'accentuent-elles lorsque le niveau d'études des parents augmente ? Nos données vont dans le sens de celles obtenues par De Meulemeester (2001) et Alaluf, Imatouchan, Marage et alii (2003) pour les étudiants universitaires de la CFB : la différence de réussite à l'université entre les garçons et les filles est d'autant plus marquée que le niveau d'études des parents est élevé, même avec des caractéristiques du parcours scolaire antérieur identiques. Toutes autres choses étant égales par ailleurs, la chance de réussite de la première année universitaire des filles est 1,5 fois plus élevée que celle des garçons lorsqu'aucun parent n'est diplômé de l'enseignement supérieur, 1,7 fois plus élevée lorsqu'au moins un parent est diplômé de l'enseignement supérieur hors universités et 1,7 fois plus élevée lorsqu'au moins un parent est diplômé de l'université.

Enfin, en guise d'ultime confirmation, nous avons analysé l'interaction entre le sexe et le niveau d'études des parents en étudiant les différences d'impact du niveau d'études des parents sur la réussite en première année universitaire selon le genre. II se confirme que la réussite des filles est plus fortement liée au niveau de diplôme des parents que celle des garçons. Avec un parcours scolaire antérieur équivalent, il est plus déterminant pour une fille que pour un garçon d'avoir un parent possédant un diplôme universitaire. De surcroît, lorsque l'on distingue le niveau de diplôme du père et de la mère, seul le niveau d'études du père a de l'influence sur la réussite des garçons alors que, chez les filles, le niveau de diplôme de la mère conserve une influence sur leur probabilité de réussite.

Comment de telles différences de réussite selon le sexe peuvent-elles s'interpréter? Si l'on s'en tient aux différences globales de réussite entre garçons et filles, celles-ci peuvent s'interpréter, comme aux autres niveaux de scolarité, par des attitudes plus favorables des filles par rapport aux études, leur plus grande implication et le fait qu'elles y consacrent davantage de temps que les garçons (Gruel \& Thiphaine, 2004). Toutefois, comme le souligne Terrail (1992a), si les filles s'investissent, s'impliquent dans leurs études, c'est " bien plus que par conformité docile à la contrainte " (Terrail, 1992a, p. 672) - une hypothèse souvent invoquée pour expliquer la meilleure réussite des 
filles. En suivant Duru-Bellat, Kieffer et Marry (2001), une piste interprétative renvoyant à une perception différenciée selon le sexe de la valeur des diplômes de l'enseignement supérieur pourrait également être formulée. Selon cette hypothèse, la réussite des études supérieures continuerait à s'inscrire dans un projet émancipatoire davantage chez les filles que chez les garçons ; l'ascension sociale et l'autonomie financière que permet un diplôme universitaire seraient ainsi davantage présentes à l'horizon des filles, qui ont encore « des places à gagner ». Dans la toute grande majorité des pays de l'OCDE, les filles aspirent en effet davantage que les garçons à accomplir des études universitaires, et ceci à performances égales dans PISA (Dupriez, Monseur \& Van Campenhoudt, 2012).

Reste à expliquer pourquoi le diplôme des parents se révèle plus déterminant pour les filles, et pourquoi, plus particulièrement, l'écart de réussite selon le sexe est d'autant plus marqué que les parents ont un diplôme élevé. Ces résultats sont en accord avec ceux d'autres travaux menés dans l'enseignement universitaire en CFB (Alaluf, Imatouchan, Marage et al., 2003 ; De Meulemeester, 2001). Le phénomène semble donc bien établi. En revanche, nos résultats s'écartent de ceux de Duru-Bellat (1995) et de Terrail (1992a, 1992b). Pour rappel, ces derniers travaux portent sur l'enseignement secondaire ; cet élément pourrait à lui seul expliquer la divergence de résultats. Par ailleurs, même si les systèmes éducatifs français et belge sont géographiquement proches, ils comportent nombre de différences, surtout à l'approche de l'enseignement supérieur, susceptibles d'expliquer que nos résultats diffèrent de ceux établis par des sociologues français. Rappelons notamment que la réussite du secondaire est sanctionnée en France par le baccalauréat, un examen perçu comme sélectif et exigeant. Rien de tel en CFB, où l'accès aux études supérieures (excepté pour les études d'ingénieur civil) est totalement libre. Par ailleurs, l'absence de grandes écoles réputées et de classes préparatoires, ainsi que la présence en CFB d'un enseignement supérieur non universitaire court fréquenté par plus de $40 \%$ des étudiants, perçu comme moins prestigieux que l'université et où se réorientent un certain nombre d'étudiants après un échec ou un abandon à l'université, donnent au paysage de l'enseignement supérieur en CFB des singularités qui le distinguent assez nettement du modèle français. Signalons en particulier qu'il n'existe pas en CFB, à l'instar de ce que l'on observe en France, une sorte de bien rare (accès aux grandes écoles et à leurs classes préparatoires) qui resterait davantage l'apanage des garçons et qui organiserait en quelque sorte tout le positionnement compétitif des garçons et des filles en amont. II existe certes une inégalité de répartition des filles et des garçons par domaine d'études à l'intérieur de l'université en CFB, conforme au scénario classique - davantage de garçons en sciences exactes et davantage de filles en sciences humaines -, mais les filles sont aussi très nombreuses dans certains domaines scientifiques comme la médecine ou la médecine vétérinaire, qui comptent parmi les études les plus prestigieuses.

À l'instar de Terrail (1992b), il nous semble fructueux de poser le problème dans les termes suivants : les garçons dont les parents sont diplômés du supérieur ou de l'université, donc en principe fortement dotés en capital culturel, réussissent moins bien et de façon caractérisée par rapport aux filles d'origine socioculturelle équivalente. Quels seraient donc les atouts dont disposeraient les filles dont les parents sont diplômés du supérieur et qui les protégeraient de l'échec en première année universitaire ? Les explications générales évoquées ci-avant (mobilisation, implication, usage de soi) ont certes ici aussi leur pertinence. Mais n'existerait-il pas des différences de genre dans les pratiques culturelles ou les représentations liées aux études universitaires qui feraient en sorte que les filles, de nos jours, et en particulier si elles viennent d'un milieu favorisé, possèdent davantage d'atouts que les garçons ?

Les données d'une recherche empirique menée en 2008 en CFB (Dupont \& Lafontaine, 2009, 2011; Maroy \& Van Campenhoudt, 2010) auprès d'un échantillon de 2147 élèves de dernière année du secondaire sur leurs aspirations d'études apportent des éléments intéressants de ce point de vue (voir l'annexe 2). Ainsi, il apparaît que, quel que soit le diplôme des parents, il est toujours plus important pour les filles que pour les garçons de faire des études supérieures. Par ailleurs, les garçons ont tendance à s'estimer capables de réussir des études universitaires plus souvent que les filles. On peut supposer que les filles abordent dès lors leurs études avec une moindre confiance, ce qui les pousse à s'investir davantage dans leurs études. Confiants dans leur capacité de réussir, les garçons, qui se disent toutefois moins confiants dans leur capacité de trouver un équilibre entre études et loisirs, se montrent probablement moins assidus. Enfin, et ceci est particulièrement important pour notre propos, le questionnaire comporte quelques items relatifs à des pratiques culturelles légitimes (au sens de Bourdieu, 1979) : fréquentation de musées ou d'expositions, de spectacles, de concerts de musique classique ou de jazz. Quels que soient le sexe et l'origine socioculturelle des étudiants 
(mesurée par le diplôme des parents), ces pratiques sont peu fréquentes. On y retrouve cependant, pour plusieurs indicateurs, un phénomène intéressant : ces pratiques deviennent plus fréquentes, chez les filles, en fonction du niveau de diplôme des parents, ce que nous n'observons pas chez les garçons. Vu sous un autre angle, les garçons en fin de secondaire ont des pratiques culturelles plus homogènes, moins différenciées, parfois indistinctes selon le diplôme des parents, tandis que chez les filles, celles dont les parents ont un diplôme universitaire déclarent des pratiques culturelles légitimes plus fréquentes que les filles dont aucun des parents ne possède un diplôme du supérieur ${ }^{17}$. Si cette analyse est correcte en matière de transmission du capital culturel, les « héritiers" d'aujourd'hui seraient, de manière privilégiée, des " héritières ". Tout ceci mérite évidemment vérifications empiriques et approfondissement ${ }^{18}$.

Par ailleurs, plusieurs recherches (Albouy \& Tavan, 2007 ; Shavit, Arum \& Gamoran, 2007 ; Merle, 1996) ont exploré I'hypothèse d'une différenciation de l'enseignement supérieur couplée au phénomène massif d'expansion observé au cours des dernières décennies. Dans des systèmes où l'offre d'enseignement supérieur s'est différenciée et stratifiée à mesure que cet enseignement se massifiait, les inégalités sociales et de genre se déplaceraient aux niveaux et segments les plus sélectifs du système. Ainsi, en France, la féminisation serait généralisée, sauf dans les classes préparatoires où les garçons continuent à être numériquement nombreux (Merle, 1996). Dans la même perspective, Albouy et Tavan (2007) ont aussi mis en évidence une polarisation sociale accrue ${ }^{19}$, pour les filles de certaines filières universitaires (le droit et la médecine notamment).

Ces études soulignent l'intérêt d'une approche qui embrasse l'ensemble de l'enseignement supérieur d'une part et qui travaille de manière fine, par domaine ou filière d'études, d'autre part. Vu les données disponibles et la taille réduite des effectifs par filière, nous n'avons pas pu assumer pleinement une telle approche dans le cadre de la présente recherche. Toutefois, les analyses exploratoires effectuées permettent de dégager certaines tendances. Ainsi il ne fait aucun doute, étant donné l'inégale répartition des garçons et des filles par domaine, que des phénomènes de sursélection académique et sociale sont en jeu pour les filles dans certains secteurs d'études. C'est dans le domaine des sciences exactes que les filles d'origine plus modeste sont les moins nombreuses et que l'impact du diplôme des parents (et de la mère en particulier) est le plus fort pour les filles. C'est aussi là, sans surprise, que l'écart de réussite entre garçons et filles est le plus marqué. L'inverse est observé en sciences humaines et sociales. Ces quelques résultats indiquent que, sur les questions de stratification et de polarisation sociale ou sexuée des domaines, les phénomènes peuvent varier d'un système éducatif à un autre, sans oublier des évolutions temporelles récentes. C'est à cette conclusion qu'aboutissent Shavit, Arum et Gamoran (2007) au terme d'une étude comparée de la stratification dans l'enseignement supérieur menée dans quinze systèmes éducatifs. Une des particularités du système d'enseignement supérieur en CFB de ce point de vue est l'absence de toute sélection ou contingentement (ouverture) et la difficulté d'affirmer avec certitude quelles seraient les études les plus prestigieuses, celles réservées à une « élite " (le prestige peut être qualifié de diffus).

Avant de conclure, arrêtons-nous un instant sur un dernier résultat. Lorsque l'on distingue l'influence respective du diplôme du père et de la mère, ce dernier n'apporte pas d'explication supplémentaire à la réussite des garçons, une fois tenu sous contrôle le diplôme du père, alors que chez les filles, le diplôme de la mère non seulement explique une partie de la réussite des filles, à diplôme du père constant, mais pèse autant, sinon davantage, que ce dernier (voir le tableau 6b). Ce résultat est à rapprocher d'une série d'éléments mis en évidence par Terrail (1992a, 1992b) notamment. Ainsi les filles parlent davantage avec leurs mères qu'avec leurs pères de leur avenir professionnel (Terrail, 1992b). Terrail souligne le « caractère complice spécifique de la relation mère/fille, particulièrement s'agissant des mères actives, mais également son rôle essentiel dans la réussite scolaire " (Terrail, 1992b, p. 87). Henri-Panabière (2010) a également mis en évidence, en étudiant le cas d'élèves en difficultés de parents fortement diplômés, "une transmission plus intense entre mères et filles qu'entre mères et fils » (Henri-Panabière, 2010, p. 472). Toujours selon Terrail, " la réussite scolaire est d'abord l'affaire de l'intéressé. Pour un enfant de cadre, elle fait partie de l'identité sociale que sa naissance lui propose. [...] Accéder aux études supérieures, c'est emprunter une voie que ses parents ont déjà parcourue [...]. Elle n'a pas ce naturel dans les milieux populaires » (Terrail, 1992b, p. 84). Le même type de raisonnement peut à notre avis être étendu aux filles. Pour les jeunes femmes d'aujourd'hui qui entreprennent des études universitaires, en particulier dans le domaine des sciences qui reste peu féminisé, le fait que leur mère ait "ouvert la voie " pèse de tout son poids, vu le dialogue singulier existant entre mères et filles. 


\section{CONCLUSION ET PERSPECTIVES}

Des modèles d'analyse de régression multivariés ont été appliqués à une base de données de 2927 étudiants de la Communauté française de Belgique entrés à l'université en 2001. Cette étude met en évidence une meilleure réussite des filles, qui ne s'explique pas par les caractéristiques du parcours scolaire antérieur. À âge, filière et options équivalents, les filles ont 1,6 fois plus de chances que les garçons de réussir leur $1^{\text {re }}$ année universitaire. Au delà de cette différence moyenne de réussite selon le sexe, des effets d'interaction entre le sexe et le niveau d'études des parents se font jour. Ainsi les écarts de réussite selon le sexe, dans tous les cas en faveur des filles, se creusent à mesure que le diplôme des parents s'élève. Il apparaît comme plus déterminant pour les filles d'avoir un parent diplômé de l'enseignement supérieur ou universitaire que ça ne l'est pour les garçons. Par ailleurs, si l'on distingue l'influence respective du diplôme du père et de la mère, il ressort des analyses que le diplôme du père est significativement lié à la réussite des étudiants, quel que soit leur sexe. En revanche, alors que le diplôme de la mère n'est pas significativement lié à la réussite des garçons à diplôme du père équivalent, il l'est dans le cas des filles : à diplôme du père constant, les filles dont la mère est diplômée de l'université réussissent significativement mieux que celles dont la mère ne possède pas de diplôme universitaire.

Ces différences de genre et ces effets croisés du genre et de l'origine sociale ont été interprétés en se référant aux travaux de Duru-Bellat, Kieffer et Marry (2001) ainsi que de Terrail (1992a, 1992b) sur la mobilisation scolaire des filles et en faisant l'hypothèse que la réussite à l'université peut représenter, aux yeux des filles de ce début de $\mathrm{XXI}^{\mathrm{e}}$ siècle, un enjeu plus important que pour leurs homologues masculins, ce qui justifierait leur plus grand investissement dans les études. Par ailleurs, les données d'une enquête par questionnaire menée en 2009 auprès d'un échantillon important d'élèves fréquentant la dernière année de l'enseignement secondaire en CFB ont permis de mettre en évidence une plus grande homogénéité des pratiques culturelles légitimées (au sens de Bourdieu, 1979) chez les garçons que chez les filles. Ainsi la fréquentation de musées, d'expositions, de spectacles ou encore de concerts de jazz et de musique classique augmente, pour les filles, en fonction du niveau de diplôme des parents, ce que nous n'observons pas chez les garçons (voir l'annexe 2). L'héritage du capital culturel dans les familles davantage dotées aurait donc quelques "ratés » du côté des garçons, alors que du côté des filles, la transmission continuerait à se faire comme par le passé. Cette hypothèse interprétative demanderait évidemment à être confirmée et étudiée plus en profondeur dans des travaux consacrés spécifiquement à cet objet.

Par ailleurs, la présente étude comporte un certain nombre de limites qu'il convient de souligner au moment de conclure. La base de données utilisée est la plus large que l'on puisse à l'heure actuelle mobiliser pour la CFB, dans la mesure où les bases de données complètes disponibles ne comportent pas de données sociodémographiques. Le nombre d'étudiants de l'échantillon est donc limité et provient seulement de deux institutions universitaires. Les comparaisons effectuées avec les données du CREF (couvrant l'ensemble de la population universitaire), quand elles étaient possibles, ont montré, et c'est rassurant, que les résultats en matière de réussite selon le sexe étaient très proches (dans la base de données utilisée pour nos analyses et dans les données de population du CREF). En outre, comme nous l'avons répété, il s'agit de données relatives à la CFB et les résultats mis en évidence portent la marque de ce contexte. II serait intéressant de voir si le même type d'influence croisée entre le sexe et l'origine socioculturelle s'observe dans d'autres systèmes éducatifs. Enfin cet article s'est centré sur la réussite à l'issue de la première année universitaire. II conviendrait de voir si le même type d'influence croisée entre le sexe et le niveau d'études des parents se reproduit pour d'autres années d'études universitaires ${ }^{20}$, et surtout dans l'enseignement supérieur non universitaire21. Au regard des enjeux de réduction des inégalités, il est en effet malaisé de raisonner sur la seule université - le segment le plus prestigieux de l'enseignement supérieur en CFB - sans disposer d'un éclairage du côté de l'enseignement supérieur non universitaire.

Dominique Lafontaine dlafontaine@ulg.ac.be Université de Liège (Belgique), Analyse des systèmes et pratiques d'enseignement

Vincent Dupriez Université catholique de Louvain (Belgique), GIRSEF

Maud Van Campenhoudt Université catholique de Louvain (Belgique), GIRSEF

Catherine Vermandele Université libre de Bruxelles (Belgique), laboratoire de méthodologie du traitement des données 


\section{NOTES}

1 Étudiants entrant pour la première fois à l'université.

2 Le CREF est le conseil des recteurs des universités de la Communauté française de Belgique. Les bases de données du CREF, il faut le préciser, ne comportent pas de données sociologiques (profession ou niveau d'études des parents).

3 Les élèves issus de l'enseignement professionnel ne peuvent accéder directement à l'université.

4 II s'agit de modèles logistiques estimés sur la base d'un échantillon de 2531 étudiants s'inscrivant pour la première fois en première année à l'ULB en 1997 ou en 2001.

5 Mentionnons cependant l'étude d'Albouy et Tavan (2007) qui ont étudié systématiquement la démocratisation selon le niveau de diplôme des parents et le sexe des étudiants. Toutefois leur recherche sur la polarisation sociale par filière porte sur des générations déjà anciennes (de 1950 à 1974).

6 Le milieu social étant dans ce cas mesuré par la CSP du père et de la mère, non par leur diplôme.

7 Nous n'avons hélas pas pu identifier d'étude en France croisant, avec des modèles statistiques appropriés, le sexe et l'origine sociale et portant sur la réussite post-secondaire. On mentionnera cependant Lemistre (2006) qui, en travaillant de manière descriptive, conclut que " la profession de chaque parent a un impact moyen "relativement" proche pour les filles et les garçons ; même si ces derniers retirent parfois un petit avantage de la profession de leurs parents " (Lemistre, 2006, p. 70).

8 Dans une revue de la question, Buchmann, DiPrete et McDaniel (2008) soulignent de leur côté que " la relation entre l'origine familiale et la réussite des études universitaires a changé pour les hommes et les femmes dans la seconde moitié du xxe siècle. [...] Ce changement a produit une situation où l'avantage des filles a d'abord émergé dans les familles où les pères sont absents ou peu éduqués. L'écart reste le plus large parmi ces familles, mais s'est graduellement étendu à tous les types de familles " (Buchmann, DiPrete \& McDaniel, 2008, p. 327).

9 Ceci fait d'ailleurs de la Belgique francophone une terre particulièrement prisée par les étudiants des pays limitrophes qui pratiquent le contingentement. Dans certaines filières, comme pour les études vétérinaires, la proportion d'étudiants français est très importante, jusqu'à $85 \%$ à l'Université de Liège par exemple.

10 La ventilation des étudiants par sexe et par diplôme des parents, selon le secteur d'études, figure en annexe 1.

11 Ces 2927 individus représentent 35,8 \% des nouveaux étudiants dans ces deux institutions. II n'était pas envisageable, dans le cadre de la présente recherche, d'effectuer une enquête sur un échantillon représentatif, d'autant que l'un des objectifs du travail était de mesurer des évolutions temporelles. Le choix a donc été fait d'utiliser les bases de données disponibles, ce qui limite le nombre de variables et la couverture de l'ensemble des institutions, qui sont au nombre de cinq pour l'ensemble de la CFB (dont trois universités offrant toutes les options).

12 Rappelons que la définition de ces modalités ne constitue pas un choix des auteurs. II s'agit des données existant dans la base de données.
13 Dans le secteur des sciences de la santé, contrairement aux deux autres secteurs d'études, le test $\chi^{2}$ d'indépendance entre la réussite et le sexe conduit au non-rejet de l'hypothèse d'indépendance $(\mathrm{V}$ de Cramer $=0,061 ; p=0,183$ ).

14 Ce coefficient d'association est étroitement lié à la statistique utilisée dans le test $\chi^{2}$ d'indépendance.

15 C'est dans le secteur des sciences de la santé que l'influence du diplôme des parents est la plus marquée $(1,9$ fois plus de chances de réussite si un des parents possède un diplôme universitaire) et dans le secteur des sciences humaines et sociales qu'elle est la plus basse (1,5 fois plus de chances).

16 Comme le montre l'annexe 1, la manière dont garçons et filles se répartissent par secteur d'études, en fonction du diplôme des parents, est particulière et asymétrique. Ainsi la proportion de garçons dont au moins un des parents est diplômé de l'université et de garçons dont aucun parent n'a ce type de diplôme est stable et tourne autour de $50 \%$ quel que soit le secteur. En revanche, pour les filles, la situation est différente. En sciences, le taux de filles dont un des parents est diplômé de l'université est de $57 \%$, ce qui confirme que les filles inscrites en sciences sont sursélectionnées. En sciences humaines et sociales, les filles de parents diplômés de l'université représentent $40 \%$ des filles. En sciences de la santé, elles sont $41 \%$.

17 À noter que pour d'autres pratiques, telles que la lecture du journal et les discussions en famille autour de sujets politiques et sociaux, les différences classiques en fonction de l'origine socioculturelle des parents sont observées, pour les garçons comme pour les filles.

18 Les travaux sur la transmission culturelle aboutissent à des constats qui vont dans le même sens. Ainsi, Octobre, Détrez, Mercklé et alii (2011), qui ont mené une étude longitudinale sur la diversification des formes de transmission culturelle, soulignent que « la féminisation tendancielle des habitus cultivés est une mutation bien identifiée " (Octobre, Détrez, Mercklé et al., 2011, p. 79).

19 Pour des cohortes d'étudiants nés entre 1950 et 1974, rappelonsle.

20 Des analyses menées sur la répétition de la première année suite à un échec indiquent que la décision de redoubler plutôt que de quitter l'université ne varie pas selon le sexe. En revanche, elle est liée au diplôme des parents chez les garçons uniquement. Les filles qui recommencent la première année d'université la réussissent mieux que les garçons (écart de $15 \%$ de réussite), ainsi que les jeunes dont un des parents possède un diplôme universitaire. L'interaction n'a pas été testée dans ce cas (Vermandele, Dupriez, Maroy et al., 2012).

21 Une chose semble clairement établie : le taux de réussite des filles est également meilleur dans l'enseignement supérieur de type court (écart de $15 \%$ ) et de type long (écart de $12 \%$, voir Ministère de la Communauté française de Belgique, 2010). Les indicateurs sont établis au départ de cohortes complètes d'étudiants; les bases de données ne comportent hélas pas de données sociodémographiques. 


\section{BIBLIOGRAPHIE}

ALALUF M., IMATOUCHAN N., MARAGE P. et al. (2003). Les filles face aux études scientifiques. Réussite scolaire et inégalités d'orientation. Bruxelles : Éd. de l'Université de Bruxelles.

ALBOUY V. \& TAVAN C. (2007). « Accès à l'enseignement supérieur en France : une démocratisation réelle mais de faible ampleur ". Économie et statistique, no 410, p. 3-22.

ARIAS ORTIZ E. \& DEHON C. (2008). «What are the factors of success at university? A case study in Belgium ". CESifo Economic studies, vol. 54, no 2, p. 121-148.

BAUDELOT C. \& ESTABLET R. (1992). Allez les filles ! Paris : Éd. du Seuil.

BOURDIEU P. (1979). La distinction. Paris : Éd. de Minuit.

BREEN R., LUIJKS R., MÜLLER W. \& POLLAK R. (2010). "Long-term trends in educational inequality in Europe: Class inequalities and gender differences ". European Sociological Review, vol. 26, n॰1, p. 31-48.

BUCHMANN C., DiPRETE T. \& McDANIEL A. (2008). "Gender inequalities in education ". Annual Review of Sociology, vol. 34, p. 319-337.

COMMUNAUTÉ FRANÇAISE DE BELGIQUE (2010). Annuaire de l'enseignement de plein exercice et budget des dépenses d'enseignement. Bruxelles : Communauté française de Belgique, service des statistiques de I'ETNIC. En ligne : http://www. statistiques.cfwb.be/index.php?id=518 (consulté le 15 juin 2012).

CREF (2003). Les étudiants et le personnel des institutions universitaires francophones de Belgique. Bruxelles: CREF. En ligne : http://www.cref.be/Annuaire_2003. htm (consulté le 15 juin 2012).

DE KERCHOVE A. \& LAMBERT J.-P. (2001). " Choix des études supérieures et motivations des étudiant(e)s $"$. Reflets et perspectives de la vie économique, vol. 40 , n० 4, p. 41-55.

DE MEULEMEESTER L. (2001). La démocratisation de l'enseignement universitaire : mythe ou réalité ? Mémoire présenté en vue de l'obtention du grade de licenciée en sociologie, Université catholique de Louvain, Belgique.

DE MEULEMEESTER L. \& ROCHAT L. (2003). “ Le développement de l'enseignement supérieur assuret-il le développement économique ? Une approche cliométrique ". Économies et sociétés, vol. 29, no 1 , p. 43-73.

DONNI O. \& PESTIEAU P. (1995). «Peut-on parler de démocratisation de l'enseignement supérieur ? " Reflets et perspectives de la vie économique, vol. 34, p. 415-427.

DROESBEKE J.-J., HECQUET I. \& WATTELAR C. (2001). La population étudiante. Description, évolution, perspectives. Paris: Ellipses.

DROESBEKE J.-J., LECRENIER C., TABUTIN D. \& VERMANDELE C. (2008). Réussite ou échec à l'université. Trajectoires des étudiants en Belgique francophone. Bruxelles : Éd. de l'Université de Bruxelles.
DUPONT V. \& LAFONTAINE D. (2009). «Les jeunes qui vont travailler dès la fin de l'enseignement secondaire : profil et motivations ». Scientia Paedagogica Experimentalis, vol. 46, no 1, p. 121-144.

DUPONT V. \& LAFONTAINE D. (2011). « Les choix d'études supérieures sont-ils liés à l'établissement secondaire fréquenté ? "Revue suisse des sciences de l'éducation, vol. 3, no 33, p. 461-478.

DUPRIEZ V., MONSEUR C. \& VAN CAMPENHOUDT $M$. (2012). " Le poids de l'origine socioculturelle des élèves et de leur environnement scolaire sur leurs aspirations d'études supérieures: les bases d'une comparaison internationale ". L'orientation scolaire et professionnelle, vol. 41, no 1, p. 29-56.

DURU-BELLAT M. (1989). L'école des filles. Quelle formation pour quels rôles sociaux? Paris : L'Harmattan.

DURU-BELLAT M. (1995). «Filles et garçons à l'école, approches sociologiques et psycho-sociales ». Revue française de pédagogie, no 110, p. 75-109.

DURU-BELLAT M., KIEFFER A. \& MARRY C. (2001). "La dynamique des scolarités des filles : le double handicap questionné ". Revue française de sociologie, vol. 42, no 2, p. 251-277.

EURYDICE (2007). Chiffres clés de l'enseignement supérieur en Europe. Bruxelles : Eurydice.

GRUEL L. \& THIPHAINE B. (2004). Des meilleures scolarités féminines aux meilleures carrières masculines. Rapport de recherche. Paris: Observatoire de la vie étudiante.

HENRI-PANABIÈRE G. (2010). "Élèves en difficultés de parents fortement diplômés ". Sociologie, vol. 1, n० 4, p. 457-477.

LEMISTRE P. (2006). "Égalité des chances ou des résultats : l'influence du genre ". Formation emploi, n० 93, p. 67-77.

MAROY C. \& VAN CAMPENHOUDT M. (2010). " Démocratisation de l'enseignement supérieur en Belgique francophone : le poids de l'autosélection et des familles ». Éducation et sociétés, vol. 26, n², p. 89-106.

MERLE P. (1996). « Les transformations sociodémographiques des filières de l'enseignement supérieur de 1985 à 1995. Essai d'interprétation ». Population, vol. 51, n 6, p. 1181-1210.

MINISTĖRE DE LA COMMUNAUTÉ FRANÇAISE DE BELGIQUE (2008). Les indicateurs de l'enseignement 2008. Bruxelles : Ministère de la Communauté française de Belgique. En ligne : http:// www.enseignement.be/index.php?page $=26930$ (consulté le 15 juin 2012).

MINISTÈRE DE LA COMMUNAUTÉ FRANÇAISE DE BELGIQUE (2010). Les indicateurs de l'enseignement 2010. Bruxelles : Ministère de la Communauté française de Belgique. En ligne : http:// www.enseignement.be/index.php?page $=26287$ (consulté le 15 juin 2012).

MINISTÈRE DE LA COMMUNAUTÉ FRANÇAISE DE BELGIQUE (2011). Les indicateurs de l'enseignement 2011. Bruxelles : Ministère de la Communauté française de Belgique. En ligne : http:// 
www.enseignement.be/index.php?page $=26464$ (consulté le 15 juin 2012).

NEUVILLE S., FRENAY M. \& BOURGEOIS É. (2007). “Task value, self-efficacy and goal orientations: Impact on self-regulated learning, choice and performance amoung university students ". Psychologica Belgica, vol. 47 , no $1 / 2$, p. 95-117.

OCDE (2010). Regards sur l'éducation. Paris : OCDE.

OCTOBRE S., DÉTREZ C., MERCKLÉ P. \& BERTHOMIER N. (2011). " La diversification des formes de la transmission culturelle : quelques éléments de réflexion à partir d'une enquête longitudinale sur les pratiques culturelles des adolescents ". Recherches familiales, n० 8, p. 71-80.

REAY D., DAVID M. \& BALL D. (2005). Degrees of choice: Social class, race and gender in higher education. Londres: Trentham Books.

SHAVIT Y., ARUM R. \& GAMORAN A. (2007). Stratification in higher education. A comparative study. Palo Alto : Stanford University Press.

TERRAIL J.-P. (1992a). " Destins scolaires de sexe: une perspective historique et quelques arguments ". Population, vol. 47, n$^{\circ} 3$, p. 645-676.
TERRAIL J.-P. (1992b). «Réussite scolaire : la mobilisation des filles ". Sociétés contemporaines, no 11-12, p. 5389.

TINTO V. (1982). « Limits of theory and practice in student attrition ". Journal of Higher Education, vol. 53, n॰ 6 , p. 687-700.

TINTO V. (1997). "Classrooms as communities». Journal of Higher Education, vol. 68, n॰ 6, p. 599-623.

VALLET L.-A. (2010). «Expansion des systèmes éducatifs et dynamique des inégalités sociales devant l'enseignement : quelques jalons de la recherche comparative en sociologie ". Économie et statistique, no 433-434, p. 23-29.

VERMANDELE C., DUPRIEZ V., MAROY C. \& VAN CAMPENHOUDT M. (2012). " Réussir à l'université : I'influence persistante du capital culturel de la famille ". Les cahiers de recherche du GIRSEF, no 87.

VERMANDELE C., PLAIGIN C., DUPRIEZ V., MAROY C., VAN CAMPENHOUDT M. \& LAFONTAINE D. (2010). "Profil des étudiants entamant des études universitaires et analyse des choix d'études ". Les cahiers de recherche en éducation et formation, n० 78 , p. 3-58.

\section{ANNEXE 1. RÉPARTITION DES ÉTUDIANTS SELON LE DIPLÔME DES PARENTS ET LE SEXE, PAR SECTEUR D'ÉTUDES}

\begin{tabular}{|l|c|c|}
\cline { 2 - 3 } \multicolumn{1}{c|}{} & $\begin{array}{c}\text { Aucun parent diplômé } \\
\text { de l'université }\end{array}$ & $\begin{array}{c}\text { Au moins un parent diplômé } \\
\text { de l'université }\end{array}$ \\
\hline Tous trois secteurs confondus & & 1333 \\
\hline Garçons & 627 & 633 \\
\hline Filles & 967 & 700 \\
\hline $\begin{array}{l}\text { Sciences humaines et sociales (dont lettres, } \\
\text { droit, sciences économiques et de gestion) }\end{array}$ & & \\
\hline Tous & 1119 & 880 \\
\hline Garçons & 401 & 398 \\
\hline Filles & 718 & 482 \\
\hline \multicolumn{1}{|c|}{ Sciences (hors sciences de l'ingénieur) } & 215 & 243 \\
\hline Tous & 148 & 153 \\
\hline Garçons Sciences de la santé & 67 & 90 \\
\hline Filles & & 210 \\
\hline & 260 & 82 \\
\hline Tous & 78 & 128 \\
\hline Garçons & 182 & \\
\hline Filles & & \\
\hline
\end{tabular}


Voici les résultats d'une enquête menée en 2009 auprès d'un échantillon de 2147 jeunes de la Communauté française de Belgique fréquentant une $6^{e}$ année secondaire donnant accès à l'enseignement supérieur, issus de 65 écoles différentes (Dupont \& Lafontaine, 2011). Le tableau présenté ci-après concerne le sous-échantillon des jeunes déclarant aspirer à faire des études universitaires l'année suivante. II se compose de 768 jeunes (353 garçons et 415 filles).

\begin{tabular}{|c|c|c|c|c|c|c|}
\hline & \multicolumn{2}{|c|}{$\begin{array}{c}\text { Aucun parent diplômé } \\
\text { du supérieur }\end{array}$} & \multicolumn{2}{|c|}{$\begin{array}{c}\text { Au moins un } \\
\text { parent diplômé } \\
\text { du supérieur, mais pas } \\
\text { de l'université }\end{array}$} & \multicolumn{2}{|c|}{$\begin{array}{l}\text { Au moins un parent } \\
\text { diplômé de l'université }\end{array}$} \\
\hline & Filles & Garçons & Filles & Garçons & Filles & Garçons \\
\hline $\begin{array}{l}\text { Proportion d'élèves pour } \\
\text { qui c'est plutôt ou tout à fait } \\
\text { important de faire des études } \\
\text { supérieures }\end{array}$ & $100 \%$ & $96 \%$ & $100 \%$ & $97 \%$ & $100 \%$ & $97 \%$ \\
\hline $\begin{array}{l}\text { Proportion d'élèves qui se } \\
\text { pensent capables de réussir } \\
\text { des études universitaires }\end{array}$ & $86 \%$ & $85 \%$ & $91 \%$ & $96 \%$ & $94 \%$ & $97 \%$ \\
\hline $\begin{array}{l}\text { Proportion d'élèves qui se } \\
\text { pensent capables de trouver un } \\
\text { équilibre entre études et loisirs }\end{array}$ & $76 \%$ & $65 \%$ & $73 \%$ & $66 \%$ & $77 \%$ & $62 \%$ \\
\hline $\begin{array}{l}\text { Proportion d'élèves qui disent } \\
\text { qu'il est important pour } \\
\text { leurs parents qu'ils aillent } \\
\text { à l'université }\end{array}$ & $61 \%$ & $65 \%$ & $59 \%$ & $60 \%$ & $71 \%$ & $71 \%$ \\
\hline $\begin{array}{l}\text { Proportion d'élèves qui disent } \\
\text { que leurs parents les estiment } \\
\text { capables de réussir des études } \\
\text { universitaires }\end{array}$ & $94 \%$ & $89 \%$ & $97 \%$ & $91 \%$ & $96 \%$ & $95 \%$ \\
\hline $\begin{array}{l}\text { Proportion d'élèves qui } \\
\text { fréquentent régulièrement des } \\
\text { expositions/musées }\end{array}$ & $11 \%$ & $11 \%$ & $13 \%$ & $13 \%$ & $18 \%$ & $11 \%$ \\
\hline $\begin{array}{l}\text { Proportion d'élèves qui vont } \\
\text { régulièrement à des spectacles }\end{array}$ & $15 \%$ & $12 \%$ & $24 \%$ & $14 \%$ & $26 \%$ & $11 \%$ \\
\hline $\begin{array}{l}\text { Proportion d'élèves qui vont } \\
\text { régulièrement à des concerts } \\
\text { de musique classique ou } \\
\text { de jazz }\end{array}$ & $2 \%$ & $9 \%$ & $9 \%$ & $4 \%$ & $8 \%$ & $7 \%$ \\
\hline $\begin{array}{l}\text { Proportion d'élèves qui vont } \\
\text { régulièrement à d'autres } \\
\text { concerts (musique du monde, } \\
\text { rock, rap...) }\end{array}$ & $14 \%$ & $18 \%$ & $24 \%$ & $19 \%$ & $20 \%$ & $20 \%$ \\
\hline $\begin{array}{l}\text { Proportion d'élèves qui lisent } \\
\text { régulièrement le journal }\end{array}$ & $28 \%$ & $38 \%$ & $39 \%$ & $42 \%$ & $29 \%$ & $48 \%$ \\
\hline
\end{tabular}

